\title{
The combination of endoglin and FIB-4 increases the accuracy of detection of hepatic fibrosis in chronic hepatitis $\mathrm{C}$ patients
}

\author{
Dawlat Salem $^{1}$, Magdy El-Serafy ${ }^{2}$, Eman Obeida $^{1}$, Wafaa Al-Akel${ }^{2}$, Maissa El-Raziki ${ }^{2}$, Dina Attia ${ }^{3}$, \\ Mostafa Hassan ${ }^{1}$ \\ ${ }^{1}$ Biochemistry Department, Faculty of Medicine, Cairo University, Cairo, Egypt \\ ${ }^{2}$ Endemic Medicine Department, Faculty of Medicine, Cairo University, Cairo, Egypt \\ ${ }^{3}$ Endemic Medicine Department, Faculty of Medicine, Beni-Suef University, Beni-Suef, Egypt \\ Email: dinaismail76@yahoo.com
}

Received 4 December 2011; revised 21 February 2012; accepted 4 March 2012

\begin{abstract}
Background and aim: In patients infected with chronic hepatitis $\mathrm{C}$ virus, liver biopsy is the gold standard method of staging fibrosis. Different combinations of serum markers attempted to correlate with hepatic fibrosis in place of liver biopsy and have shown encouraging results. The aim of our study is to evaluate the diagnostic value of endoglin and FIB-4 as noninvasive markers of hepatic fibrosis in HCV patients. Methods: We estimated serum endoglin \& FIB-4 index in 40 infected chronic hepatitis $C$ patients. Histological staging of hepatic fibrosis was done according to the METAVIR scoring system. Results: Both endoglin and FIB-4 index showed positive correlation with age and aspartate transaminase and inverse correlation with albumin. The diagnostic performance determined by AUROCs for early fibrosis $(\leq \mathrm{F} 2)$, was 0.868 for endoglin and 0.887 for FIB-4, at cut off values of $5.5 \& 0.98$ with sensitivity of $64.3 \% \& 82.1 \%$, and specificity of $100 \% \& 85 \%$ respectively. For advanced fibrosis $(>F 2)$, the AUROC was 0.98 for endoglin and 0.967 for FIB-4, obtained at cut off values of $6.29 \& 1.6$, with sensitivity of $100 \% \& 91.7 \%$, and specificity of $\mathbf{8 9 . 3 \%} \& \mathbf{9 2 . 9 \%}$, respectively. Conclusion: Both serum endoglin and FIB-4 index are fairly accurate in differentiating stages of hepatic fibrosis; their combination in a single equation enhanced the accuracy of fibrosis detection in chronic $\mathrm{HCV}$ patients.
\end{abstract}

Keywords: Hepatitis C Virus; Hepatic Fibrosis; Liver Biopsy; Non-Invasive Marker; Endoglin; FIB-4

\section{INTRODUCTION}

Chronic hepatitis $\mathrm{C}(\mathrm{CHC})$ is recognized as a major healthcare problem with a worldwide prevalence of over 200 million [1] with a high prevalence in Egypt. CHC is characterized by hepatic fibrosis which may progress to cirrhosis. Clinical management is dependent on the extent of liver fibrosis. Clinicians require accurate information about the degree of fibrosis to guide management decisions, predict outcome and monitor disease [2].

Liver biopsy has been the reference test for the assessment of hepatic fibrosis, but has limitations [3]. Thus, accurate and cost-effective non-invasive methods as serum markers may allow dynamic calibration of fibrosis to assess disease severity and monitor $\mathrm{CHC}$ patients \& offer an attractive alternative to liver biopsy [4].

Studies showed that available biological tests, from the simplest APRI to the most sophisticated Fibrotest or Fibrometer, had global diagnostic performances similar to each other with AUROC around 0.80 for the diagnosis of significant fibrosis [5]. These tests are clearly not accurate enough to precisely stage liver fibrosis in all cases [2]. However, they may give important clues on fibrosis especially when used in combination [6]. If markers with high predictive values of fibrosis can be obtained, fewer liver biopsies would need to be done and thus the cost and risk of liver biopsy would be lessened [3]. Endoglin is a component of the TGF- $\beta$ receptor system, which is predominantly expressed in vascular EC, and is considered as a TGF- $\beta$ type III receptor (T $\beta$ R-III) [7]. Transforming growth factor (TGF)- $\beta$ is a multifunctional cytokine that plays a central role in liver fibrogenesis [8]. Studies showed that there is increasing evidence that endoglin may play an important role in fibrogenesis, as overexpression of this protein in biopsies from patients with renal and liver fibrosis has been observed [9]. However, it doesn't has improvement neither in liver function nor in human liver fibrosis after Bile duct ligation induced in rats [10]. A possible role for endoglin is 
suggested, as a novel complementary biomarker to detect the risk of development of HCC in cirrhotic patients [11]. FIB-4 index is a simple, inexpensive, noninvasive test, which combines standard biochemical values (platelets, ALT, AST) and age to determine the degree of hepatic fibrosis [12].

The value of FIB-4 index may be because of it is ease of use, simple calculations, inexpensive, quick and need no standardization. Results are available immediately, during the outpatient visit. It is of particular importance in developing countries with limited resources. It has a higher sensitivity and specificity than most indirect markers. However, in cases with very young age and/or unexplained thrombocytosis, the degree of hepatic fibrosis may be underestimated by FIB-4 index [12].

\section{METHODS}

\subsection{Patients}

In a cross sectional study, 40 chronic infected HCV Egyptian patients were recruited from the Hepatology outpatient clinic, Al Qahira El Fatimeya Hospital. After an informed consent, a clinical and biochemical profiles were assessed. Patients were excluded if they have schistosomal hepatic fibrosis, HBV or HIV and other chronic liver disease. We included a second control population of 20 healthy volunteers for validation of our findings.

\subsection{Serum Markers}

Serum markers were done including serum aminotransferases (ALT \& AST), serum total and direct bilirubin, blood platelets count, albumin \& prothrombin time using the standards methods. HCV was diagnosed by detecting $\mathrm{HCV}$ antibody using the third generation immunoassay (EIA). Estimation of serum endoglin/CD105 using the (ELISA) R\&D Systems, Inc. USA. The FIB-4 was calculated using the following equation

$$
\begin{aligned}
& \operatorname{Age}(\text { years }) \\
& \times \operatorname{AST}[\mathbf{U} / \mathbf{L}] /\left(\text { platelets }\left[10^{9} / \mathbf{l}\right] \times(\operatorname{ALT}[\mathbf{U} / \mathbf{L}])^{1 / 2}\right)[7]
\end{aligned}
$$

ALT: Alanine aminotransferase;

AST: Aspartate aminotransferase.

\subsection{Liver Biopsy}

Histological features of liver specimens were analyzed with the METAVIR group scoring system [13]. The core biopsies were more than $10 \mathrm{~mm}$ in length and contained at least 8 portal tracts. The specimen was stained with haematoxylin eosin and Masson's trichrome for collagen. The specimen was examined by hepatopathologist blinded by the patient characteristics. Patients were classified according to their stage of fibrosis into early fibrosis (EF) $(\leq 2)$ and advanced fibrosis (AF) $(>2)$.

\subsection{Statistical Analysis}

Anova (analysis of variance) test was used to test the equality of the means of different endoglin and FIB-4. Receiver operating characteristic curves (ROC) were constructed to assess the power of the biomarkers in predicting severe and mild hepatic fibrosis by calculating the area under the curve and was considered if $>0.60$. Sensitivity, specificity, positive (PPV) and negative (NPV) predictive values were calculated for predicting early and advanced fibrosis (stages 2-4). The Chi-Square Test (goodness-of-fit) test compares the observed and expected frequencies in each category to test that all categories contain the same proportion of values. Pearson's correlation $(r)$ was done to detect the relation between all the parameters that has been studied. Univariate and multivariate logistic regressions were used to assess the association between biomarker levels and early and advanced hepatic fibrosis.

\section{RESULTS}

Out of 40 chronic infected HCV patients, the male:female ratio was $28: 12$ with male predominance $(75 \%)$ with $73.3 \%$ had early fibrosis \& $26.7 \%$ had advanced fibrosis. The females had $60 \%$ early fibrosis \& $40 \%$ advanced fibrosis. The mean age in early and advanced fibrosis was $41.86 \pm 7.5$ years $\& 48.58 \pm 6.75$ years respectively. The liver biochemical profile and the hematological features of the studied patients are shown in (Table 1).

\subsection{Results of Endoglin in Relation to Hepatic Fibrosis}

This study showed that the mean of serum endoglin le-

Table 1. Liver biochemical \& hematological profiles of the studied groups.

\begin{tabular}{lcccc}
\hline \multicolumn{1}{c}{ Liver functions } & Control $\odot \begin{array}{c}\text { Early } \\
\text { fibrosis (EF) }\end{array}$ & $\begin{array}{c}\text { Advanced } \\
\text { fibrosis (AF) }\end{array}$ & P-value \\
\hline T. Bilirubin & $0.7 \pm 0.2$ & $0.9 \pm 0.3$ & $0.9 \pm 0.5$ & 0.069 \\
Albumin & $4.4 \pm 0.4$ & $4.13 \pm 0.4$ & $3.8 \pm 0.4$ & $0.000^{*}$ \\
AST U/L (38) & $25.5 \pm 7.4$ & $42.2 \pm 13$ & $62.6 \pm 24$ & $0.000^{*}$ \\
ALT (41) U/L & $24 \pm 9.7$ & $57.1 \pm 38$ & $60 \pm 23$ & $0.002^{*}$ \\
ALP & $78 \pm 22$ & $86.4 \pm 26.9$ & $93.8 \pm 30$ & 0.262 \\
Heamatological profile & & & & \\
WBCs $\left(\times 10^{3}\right)$ & $6.7 \pm 1.1$ & $7.8 \pm 8.4$ & $6.3 \pm 1.8$ & 0.684 \\
Platelet count $\left(\times 10^{3}\right)$ & $284.2 \pm 63$ & $216.1 \pm 57$ & $175.3 \pm 48$ & $0.000^{*}$ \\
Prothrombin conc. $\%$ & $93.4 \pm 4.9$ & $84.7 \pm 9.7$ & $85.1 \pm 12.4$ & $0.005^{*}$ \\
\hline
\end{tabular}

WBCs: White Blood Cells; U/L: unit/liter; T. Bilirubin: Total Bilirubin; ALP Alkaline Phosphatase; ": Statistically significant; Values presented with mean and standard deviation. 
vels was higher in EF group than the healthy control group and higher in the AF group $(\mathrm{P}<0.001)$ (shown in Figure 1).

\subsection{Results of Endoglin in Relation to Laboratory Data}

Endoglin showed a significant positive correlation with age, AST and inverse correlation with albumin (Figure 2) in $\mathrm{HCV}$ patients.

\subsection{Results of Endoglin in Relation to FIB-4}

The Box-Plot in (Figure 3) showed that the mean of FIB-4 was highest in AF group \& higher in EF group in $\mathrm{HCV}$ patients than the healthy control group.

By comparing endoglin \& FIB-4 results, endoglin levels were concordant with FIB-4 levels $\left(\chi^{2}=43.9\right.$, $\mathrm{P}$-value $<0.001)$ (Table 2) and (Figure 4).

\subsection{Results of Endoglin \& FIB-4}

By using ROC curve of endoglin for predicting early fibrosis (EF), the best cut off value was 5.1 (Figure 5), at which sensitivity $64.3 \%$, specificity $100 \%$. Likewise for predicting advanced fibrosis (AF), the best cutoff value was 6.29 (Figure 5), at which sensitivity $100 \%$, specificity $89.3 \%$. Similarly FIB-4 predicated early fibrosis (EF) with best cutoff 0.98 , AUC was 0.887 (Figure 6), at which sensitivity $=82.1 \%$, specificity $=85 \%$. As predicting advanced fibrosis (AF), the best cutoff value was 1.6, AUC was 0.967 (Figure 6), at which sensitivity $=$ $91.7 \%$, specificity $=92.9 \%$. Table 3 summarizes the results of endoglin \& FIB-4 as markers of early \& advanced fibrosis.

\subsection{Results of Endoglin + FIB-4}

For predicting the degree of fibrosis of a given patient, endoglin concentration and FIB-4 index of this patient are utilized in a simple equation as follows:

$$
Y_{0}=\operatorname{Exp}^{[58.31-7.115(\text { endoglin })-15.75(\text { FIB- })]} \text { and }
$$

$$
Y_{1}=\operatorname{Exp}^{[28.703-3.134(\text { endoglin })-5.224(\text { FIB- } 4)]}
$$

This study showed that out of 20 control cases, only $16(80 \%)$ were correctly classified by this model and 4 were incorrectly classified as having early fibrosis. For The 28 cases with early fibrosis, 26 were correctly classified $(92.9 \%)$ by this model and 2 were incorrectly clas-

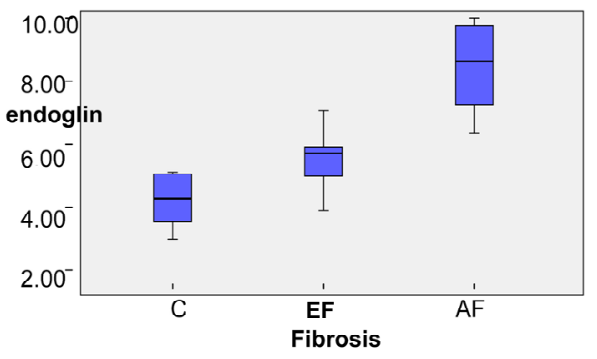

Figure 1. Box Plot of serum Endoglin concentration of the studied group.

Table 2. Shows comparison of endoglin and FIB-4 results.

\begin{tabular}{ccccc}
\hline \multicolumn{5}{c}{ FIB-4 } \\
\hline Endoglin & $<0.98$ & $0.98-1.6$ & $>1.6$ & Total \\
$<5.1 \mathrm{ng} / \mathrm{ml}$ & $19(63.33 \%)$ & $10(33.33 \%)$ & $1(3.33 \%)$ & 30 \\
$5.1-6.29 \mathrm{ng} / \mathrm{ml}$ & $2(13.33 \%)$ & $12(80 \%)$ & $1(6.67 \%)$ & 15 \\
$>6.29 \mathrm{ng} / \mathrm{ml}$ & $1(6.67 \%)$ & $3(20 \%)$ & $11(73.33 \%)$ & 15 \\
Total & 22 & 25 & 13 & 60 \\
\hline
\end{tabular}

Table 3. Comparison between endoglin and FIB-4 as markers for early \& advanced fibrosis.

\begin{tabular}{cccccccc}
\hline Endoglin AUC & $\begin{array}{c}\text { P } \\
\text { value }\end{array}$ & $\begin{array}{c}\text { Best } \\
\text { Cutoff }\end{array}$ & Sensitivity & Specificity & PPV & NPV \\
\hline EF & 0.868 & 0.0001 & 5.1 & $64.3 \%$ & $100 \%$ & $100 \%$ & $66.7 \%$ \\
AF & 0.980 & 0.0001 & 6.29 & $100 \%$ & $89.3 \%$ & $80 \%$ & $100 \%$ \\
FIB-4 & & & & & & & \\
EF & 0.887 & 0.0001 & 0.98 & $82.1 \%$ & $85 \%$ & $88.5 \%$ & $77.3 \%$ \\
AF & 0.967 & 0.0001 & 1.6 & $91.7 \%$ & $92.9 \%$ & $84.6 \%$ & $96.3 \%$ \\
\hline
\end{tabular}

PPV: Positive predictive value; NPV: Negative predictive value.
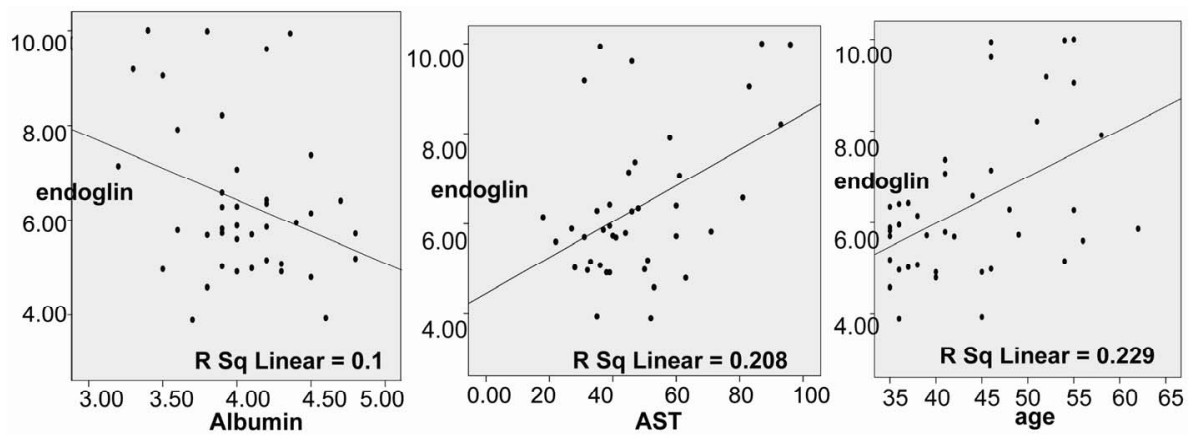

Figure 2. Positive correlation between endoglin level and each of age $(r=0.478, \mathrm{P}=0.002)$, AST $(r=0.456, \mathrm{P}=0.003)$ and albumin $(r=-0.317, \mathrm{P}=0.046)$ in HCV patients. 


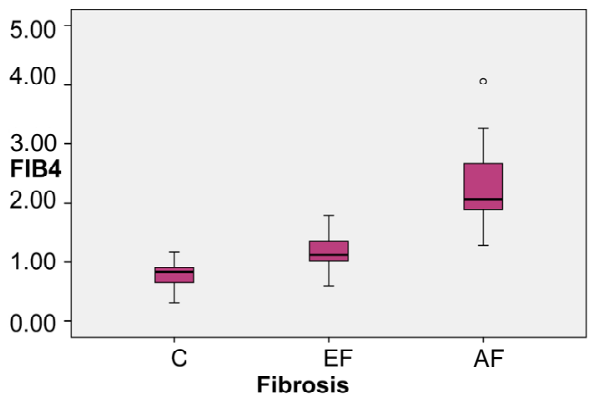

Figure 3. Box plot of FIB-4 index of the studied groups.

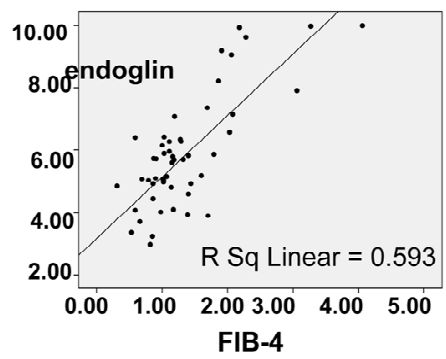

Figure 4. Positive correlation between endoglin level and FIB-4 in the studied groups $(r=0.770, \mathrm{P}=$ $0.000)$.

sified as having no fibrosis with specificity $80 \%$ \& sensitivity $92.9 \%$. Out of (12) advanced fibrosis cases, 11

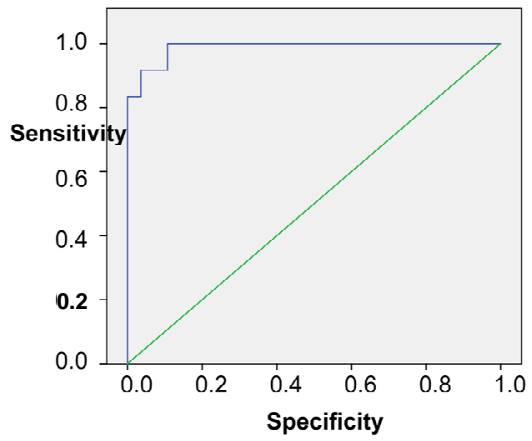

$(91.7 \%)$ were correctly classified by this model and one was incorrectly classified as having early fibrosis. The overall correct percentage by this model was $88.3 \%$ with specificity $100 \%$ \& sensitivity $91.67 \%$.

\section{DISCUSSION}

In Egypt, there is a heavy chronic liver disease burden and diagnosing severe fibrosis or cirrhosis could initiate strategies for treatment of HCV and screening for hepatocellular carcinoma. It is difficult to justify serial liver biopsies to diagnose and monitor patients with chronic $\mathrm{HCV}$ when there are limited options for managing their disease [14].

\subsection{Endoglin}

In this series, a statistically significant difference was detected between endoglin concentrations of control, EF and AF groups. This is in agreement with other studies [15].

This can be explained by the following: As endoglin is a component of the TGF- $\beta$ receptor system and the promatrix effects of TGF- $\beta$ are recognized to play a key role in the fibrotic process that characterize chronic liver diseases [11], Thus, the presence of endoglin as part of the TGF- $\beta$ receptor system could be important in determining the extent of ECM protein production by HSC following the binding of TGF- $\beta[15]$.

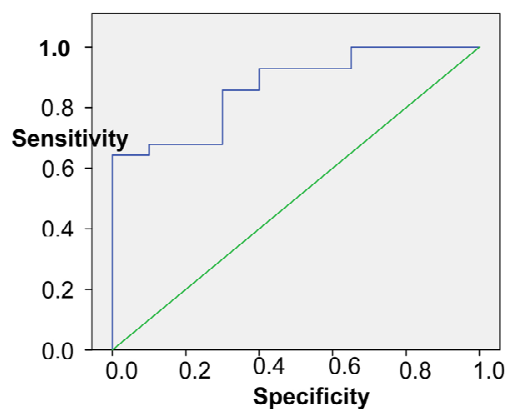

Figure 5. Shows ROC curve of endoglin for early and advanced fibrosis.
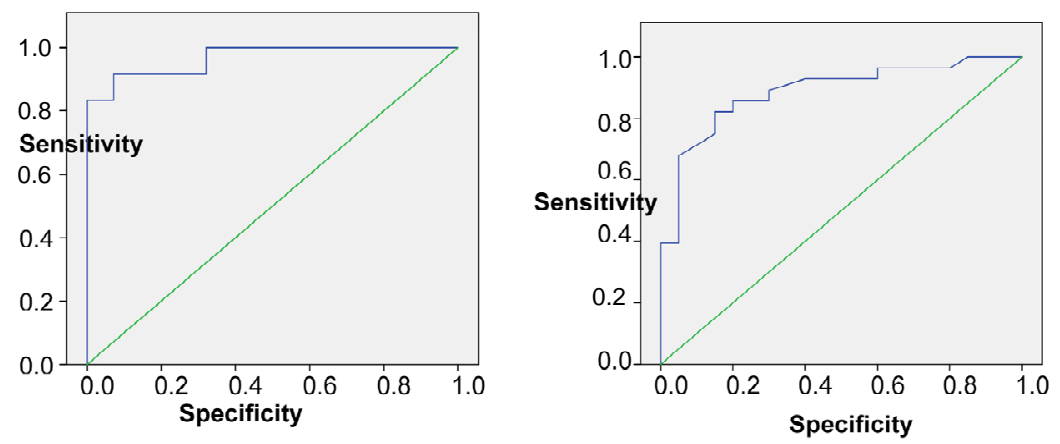

Figure 6. ROC curve of FIB-4 for early and advanced fibrosis. 
Kanzler et al. reported a close correlation between TGF- $\beta$ serum levels and the rate of fibrosis progression showing that patients with no progression of fibrosis had significantly lower TGF- $\beta$ serum levels than patients with progressive disease [16]. Other studies stated that intrahepatic expression levels of both endoglin and TGF$\beta$ increased in parallel with the histological stage of liver disease, suggesting that locally released TGF- $\beta$ may be responsible for the endoglin upregulation observed in the liver of patients with chronic HCV infection [15].

There was a statistically significant difference between endoglin and the age and AST and inverse correlation with the albumin.

For a hepatic fibrosis marker to be usable, the NPV and PPV should exceed $90 \%$ for the detection of mild and significant fibrosis [17]. The great majority of proposed biochemical markers have an AUC between 0.80 0.85 and have value not for staging the disease, but rather for differentiating early $(\leq \mathrm{F} 2)$ from advanced fibrosis ( $>$ F2 Metavir) [18].

In agreement with previous studies showing that AUC for AF was 0.789 with sensitivity of $77 \%$ and specificity of $74 \%$ in patients with chronic HCV infection [15], our study showed that Endoglin excluded significant fibrosis $(>$ F2) at cutoff 6.29 and an AUC 0.98 with a sensitivity $100 \%$ \& specificity $89.3 \%$, NPV 100\% \& PPV 80\%. Endoglin also predicted EF with a PPV 100\% and NPV $66.7 \%$.

\subsection{FIB-4}

In addition this study showed that FIB-4 index had significant statistical difference in the Control, EF and AF groups in chronic HCV patients. This was in agreement with previous studies that showed that mean FIB-4 values increased as a function of the fibrosis score and pointed to a statistically significant differences $(\mathrm{P}<0.01)$. FIB-4 index could not significantly discriminate F1 from F2 cases owing to the large overlapping of the results [7].

FIB-4 index excluded AF with AUC 0.67 at cutoff 1.6 with sensitivity $91.7 \%$ \& specificity $92.9 \%$, PPV $84.6 \%$ \& NPV 96.3\%. EF could be discriminated with AUC 0.887 at cutoff 0.98 with sensitivity $82.1 \%$ \& specificity $85 \%$, NPV $77.3 \%$ \& $88.5 \%$. This was in agreement with Vallet-Pichard et al., who reported AUC of FIB-4 for predicting AF (METAVIR F3-F4) was 0.85 and was 0.91 for cirrhosis (METAVIR F4), AUC. Also, he reported that a FIB-4 index excluded any extensive fibrosis (F3-F4) with value lower than 1.45 with a NPV 94.7\%, sensitivity of $74.3 \%$ and specificity $80.1 \%$. A FIB-4 index higher than 3.25, confirms significant fibrosis (F3-F4) with PPV $82.1 \%$, specificity $98.2 \%$ and sensitivity $37.6 \%$ [7].

\subsection{Combined FIB-4 \& Endoglin}

Stepwise combination of non-invasive markers of liver fibrosis improves the diagnostic performance in chronic hepatitis $\mathrm{C}$ and the need for liver biopsy is reduced by $50 \%-70 \%$ but cannot be completely avoided [19]. Thus the key for future studies is to determine which combination of markers is the most reliable for first-line evaluation of fibrosis in $\mathrm{HCV}$-infected patients and in what cases liver biopsy remains necessary.

In this series, we are suggesting a model in which endoglin concentration and FIB-4 index are utilized together in the same equation for predicting degree of hepatic fibrosis of a given patient. On applying this model on our cases, the overall correct percentage of classification by this model was $88.3 \%$. The sensitivity for predicting EF was $92.85 \%$ and specificity $80 \%$. For predicting AF sensitivity was $91.67 \%$ and specificity was $100 \%$. In conclusion: This study means that the combination of both endoglin levels and FIB-4, as direct \& indirect markers, in a single equation has enhanced the accuracy of fibrosis detection in chronic $\mathrm{HCV}$ patients with high sensitivity \& specificity similarly to the Fibrotest and Actitest [20,21] and the Fibrometer [22]. Furtherstudies with larger sample size are recommended to objectively charge this model and validate it for accurate assessment of the degree of hepatic fibrosis. This model presents a practical noninvasive and inexpensive alternative to liver biopsy for assessing hepatic injury in Egyptian patients with chronic $\mathrm{HCV}$ infections.

\section{REFERENCES}

[1] WHO (2002) NIH consensus statement on management of hepatitis C. Hepatology, 36, 3-20. doi:10.1053/jhep.2002.37117

[2] Parkes, J., Guha, I., Roderick, P. and Rosenberg, W. (2006) Performance of serum marker panels for liver fibrosis in chronic hepatitis C. Journal of Hepatology, 44, 462-474. doi.org/10.1016/j.jhep.2005.10.019

[3] Cadranel, J., Rufat, P. and Degos, F., (2000) Practices of liver biopsy in France: Results of a prospective nationwide survey. Journal of Hepatology, 32, 477-481. doi:10.1053/jhep.2000.16602

[4] Patel, K., Gordon, S., Jacobson, I., et al. (2004) Evaluation of a panel of non-invasive serum markers to differentiate mild from moderate-to-advanced liver fibrosis in chronic hepatitis C patients. Journal of Hepatology, 41, 935-942. doi.org/10.1016/j.jhep.2004.08.008

[5] Levoy, V., Hilleret, M.N., Sturm, N., et al. (2007) Prospective comparison of six non-invasive scores for the diagnosis of liver fibrosis in chronic hepatitis C. Journal of Hepatology, 46, 775-782. doi.org/10.1016/j.jhep.2006.12.013

[6] Westin, J., Ydreborg, M., Islam, S., et al. (2008) A noninvasive fibrosis score predicts treatment outcome in chro- 
nic hepatitis C virus infection. Scandinavian Journal of Gastroenterology, 43, 73-80. doi:10.1080/00365520701514461

[7] Vallet-Pichard, A., Mallet, V., Nalpas, B., et al. (2007) FIB-4: An Inexpensive and accurate marker of fibrosis in HCV infection. Hepatology, 46, 32-36. doi:10.1002/hep.21669

[8] The French METAVIR Cooperative Study Group (1994) Intraobserver and interobserver variations in liver biopsy interpretation in patients with chronic hepatitis C. Journal of Hepatology, 20, 15-20.

[9] Esmat, G., Metwally, M., Zalata, K., et al. (2007) Evaluation of serum biomarkers of fibrosis and injury in Egyptian patients with chronic hepatitis C. Journal of Hepatology, 46, 620-627. doi.org/10.1016/j.jhep.2006.12.010

[10] Lebrin, F., Goumans, M.J., Jonker, L., et al. (2004) Endoglin promotes endothelial cell proliferation and TGFbeta/ALK1 signal transduction. The EMBO Journal, 23, 4018-4028. doi:10.1038/si.emboj.7600386

[11] Bissell, D.M., Roulot, D. and George, J. (2001) Transforming growth factor $\mathrm{b}$ and the liver. Hepatology, 34, 859-867. doi:10.1053/jhep.2001.28457

[12] Roy-Chaudhury, P., Simpson, J.G. and Power, D.A. (1997) Endoglin, a transforming growth factor-beta-binding protein, is upregulated in chronic progressive renal disease. Experimental Nephrology, 5, 55-60.

[13] Pérez-Barriocanal, F., Docherty, N.G., Miguel Arévalo, M., et al. (2011) Endoglin upregulation in the liver after bile duct ligation in rats. Open Gastroenterology Journal, 5, 1-9. doi:10.2174/1874259901105010001

[14] Yagmur, E., Rizk, M., Stanzel, S., et al. (2007) Elevation of endoglin (CD105) concentrations in serum of patients with liver cirrhosis and carcinoma. European Journal of Gastroenterology \& Hepatology, 19, 755-761. doi:10.1097/MEG.0b013e3282202bea

[15] Clemente, M., Núňez, O., Lorente, R., et al. (2006) In- creased intrahepatic and circulating levels of endoglin, a TGF- $\beta 1$ co-receptor in patients with chronic hepatitis $\mathrm{C}$ virus infection: Relationship to histological and serum markers of hepatic fibrosis. Journal of Viral Hepatitis, 13, 625-632. doi:10.1111/j.1365-2893.2006.00733.x

[16] Kanzler, S., Baumann, M., Schirmacher, P., et al. (2001) Prediction of progressive liver fibrosis in hepatitis $\mathrm{C}$ infection by serum and tissue levels of transforming growth factor-beta. Journal of Viral Hepatitis, 8, 430-437. doi:10.1046/j.1365-2893.2001.00314.x

[17] Snyder, N., Nguyen, A., Gajula, L., et al. (2007) The APRI may be enhanced by the use of the FIBROSpect II in the estimation of fibrosis in chronic hepatitis C. Clinica Chimica Acta, 381, 119-123. doi.org/10.1016/j.cca.2007.02.046.

[18] Grigorescu, M. (2006) Noninvasive biochemical markers of liver fibrosis. Journal of Gastrointestinal and Liver Diseases, 15, 149-159.

[19] Sebastiani, G., Vario, A., Guido, M., et al. (2006) Stepwise combination algorithms of non-invasive markers to diagnose significant fibrosis in chronic hepatitis C. Journal of Hepatology, 44, 686-693. doi.org/10.1016/j.jhep.2006.01.007

[20] Imbert-Bismut, F., Ratziu, V., Pieroni, L., et al. (2001) Biochemical markers of liver fibrosis in patients with hepatitis $\mathrm{C}$ virus infection: A prospective study. Lancet, 357, 1069-1075. doi.org/10.1016/S0140-6736(00)04258-6

[21] Poynard, T., McHutchison, J., Manns, M., Rob, P. and Myers Albrecht, J. (2003) Biochemical surrogate markers of liver fibrosis and activity in a randomized trial of peginterferon alfa- $2 \mathrm{~b}$ and ribavirin. Hepatology, 38, 481492. doi:10.1053/jhep.2003.50319

[22] Cales, P., Oberti, F., Michalak, S., et al. (2005) A novel panel of blood markers to assess the degree of liver fibrosis. Hepatology, 42, 1373-1381. doi:10.1002/hep.20935

\author{
ABBREVIATIONS \\ FIB-4: Fibrosis index biochemical (Platelets, AST, ALT, \\ Age). \\ APRI: AST platelets ratio index. \\ HCV: Hepatitis $C$ virus \\ HBV: Hepatitis B virus
}

